$\diamond \curvearrowright \diamond \quad$ Full metadata for this item is available in Research@StAndrews:FullText at: http://research-repository.st-andrews.ac.uk/

\title{
Experimental field studies with non-human primates
}

Klaus Zuberbuehler

\begin{tabular}{|l|l|}
\hline Date of deposit & $28 / 11 / 14$ \\
\hline Version & This is an author version of this work. \\
\hline Access rights & $\begin{array}{l}\text { C } 2014 \text { This item is protected by original copyright. } \\
\text { This work is made available online in accordance with publisher } \\
\text { policies. This is an author version of this work which may vary } \\
\text { slightly from the published version. To see the final definitive } \\
\text { version of this paper please visit the publisher's website. }\end{array}$ \\
\hline $\begin{array}{l}\text { Citation for } \\
\text { published version }\end{array}$ & $\begin{array}{l}\text { Klaus Zuberbuehler (2014). Experimental field studies with non- } \\
\text { human primates. Current Opinion in Neurobiology, 28: } 150-156 .\end{array}$ \\
\hline $\begin{array}{l}\text { Link to published } \\
\text { version }\end{array}$ & http://dx.doi.org/10.1016/j.conb.2014.07.012 \\
\hline
\end{tabular}

NOTICE: this is the author's version of a work that was accepted for publication in Current Opinion in Neurobiology. Changes resulting from the publishing process, such as peer review, editing, corrections, structural formatting, and other quality control mechanisms may not be reflected in this document. Changes may have been made to this work since it was submitted for publication. A definitive version was subsequently published in Current Opinion in Neurobiology, 28, 2014.

DOI: $10.1016 /$ j.conb.2014.07.012 


\title{
Experimental field studies with non-human primates
}

\author{
Klaus Zuberbühler \\ Department of Comparative Cognition, University of Neuchatel, Switzerland \& \\ School of Psychology \& Neuroscience, University of St Andrews, Scotland (UK)
}

\begin{abstract}
One way to study language evolution is to compare human communication with closely related non-human primate species. This comparative approach has turned to be especially productive if subjects are studied under natural field conditions in which they have evolved. Various observation techniques have been developed, but field experiments are often needed to clarify underlying cause-effect relations. Here, I review the main experimental designs that are suitable for primate fieldwork and discuss some scientific advancements that they have generated. Field experiments are notoriously difficult to carry out for a range of reasons that are discussed.

Nonetheless, considerable progress has been made in recent years, including with great apes, which have traditionally been neglected in experimental research in the wild.
\end{abstract}

\section{Introduction}

How and why did humans evolve a communication system so radically different from other primates? An evolutionarily parsimonious scenario is that the key capacities, such as the abilities to control and combine articulatory manoeuvres, to take the audience into account, or to make inferences about the intended meaning of an utterance, have gradually evolved during human evolution and are derived from earlier forms of more primate-like communication systems. Investigating all this is not a trivial task, mainly because the fossil record generally reveals little about behavioural evolution. An alternative approach is to compare the communication behaviour of closely related modern primates; the comparative method. This requires detailed and careful field observations combined with focussed field experiments, ideally also with the great apes, our nearest living relatives.

This chapter reviews some of the scientific progress achieved by studying wild primates experimentally, including a discussion of the difficulties encountered by field workers, who address research questions this way. Lack of control over subject movements and audience composition, working with intelligent animals, and ethical issues relating to endangered species are some of the main obstacles that need to be addressed.

\section{Primate communication and human language}

Initially, researchers studying the relation of primate communication and language have largely worked in the laboratory, using artificial language systems [1]. Although this has led to substantial insights, an important concern has always been 
that subjects had been trained to respond to various requests from their human trainers in order to earn food rewards, which has raised questions about evolutionary relevance. Unless similar capacities are expressed during natural communication, results from captivity may be irrelevant for theories of language evolution and better explained as the product of human enculturation or excessive training.

The natural communication of primates has been of longstanding scientific interest, perhaps starting with fieldwork by Carpenter in the 1930s [2]*. It has quickly become clear, from this and later research, that primates possess rich, species-specific repertoires with vocal, visual, and tactile signals that have specific social functions. Studying primate communication is of course worthy in its own right but many researchers have also wondered about the relation between primate and human communication and especially language. One relevant early finding came from fieldwork with vervet monkeys, which were observed to produce a range of acoustically distinct alarm calls to different predators [3]. Historically also important was a methodological breakthrough provided by a field study on gray-cheeked mangabeys in Uganda, which demonstrated to a wide scientific audience that playback techniques could be used to investigate the function of primate signals [4]*. Although playbacks had been used before with primates, possibly first by Garner in 1890 [5], the mangabey study demonstrated that the technique was suitable to address major questions in the field. Subsequently, Seyfarth et al. [6]* then carried out a first systematic playback study with fully habituated vervet monkeys to test whether their alarm calls conveyed something about the type of predator encountered by the caller. The striking finding was that subjects responded to the different alarm call playbacks as if the corresponding predator were present, which indicated that these calls conveyed very specific meanings. This landmark study thus provided the first conclusive evidence that non-human primates possessed communicative abilities to refer to external events with recipients capable of extracting meaning from the calls. Non-human primates, it was concluded, are capable of semantic communication, not fundamentally different from how humans extract meaning from speech utterances.

This early success was followed by a substantial, experimentally based research programme on free-ranging vervet monkeys, which revealed that the mental and social world of these primates was much richer than anyone could have imagined from mere behavioural observations [7]**. For example, it could be shown that monkeys recognised each other individually by their vocalisations [8], that some calls consisted of acoustic variants with distinct functions [9], that individuals were more willing to aid unrelated individuals if those individuals have behaved affinitively towards them in the past [10], or that individuals who had learned to ignore one call type by an unreliable signaller subsequently also ignored other calls by the same individual [11].

Playback techniques soon became a popular research tool for primate fieldworkers to address a range of biological questions, which resulted in a large literature by the end of the $20^{\text {th }}$ century, including studies on intergroup interactions in titi monkeys [12], gibbons [13], howler monkeys [14] and pig-tailed macaques [15]; long-distance communication in orang-utans [16], mating strategies in gibbons [17], [18], [19] and Barbary macaques [20], call perception and categorisation in Japanese and Barbary macaques [21] [22]; call exchanges in Japanese macaques [23]; alarm calling in lemurs [24], [25], diana monkeys [26], Barbary macaques [27], bonnet macaques [28]; polyspecific grouping and anti-predator behaviour in forest monkeys [29], [30], [31]; social cognition in baboons [32]; mental representations in diana monkeys [33], [34,35]; individual recognition in Barbary macaques [36], rhesus 
macaques [37], [38] and spider monkeys [39], [40]); foraging behaviour in graycheeked mangabeys ([41]); or inter-specific communication in macaques [42] and guenons [43]. This development has continued almost exponentially into the new millennium with an increasing number of species being tested with experimental techniques with one interesting exception, the great apes. The reasons for this will be discussed below.

\section{Experimental designs}

Before planning a field experiment, researchers typically spend considerable time describing the natural behaviour patterns using traditional, well established observation methods [44]. Once the main patterns become visible, a number of choices are available to test the causal structure of the data with specific hypotheses.

A general point to consider for any experimental intervention is the quality and authenticity of the stimuli. Ideally, subjects should not realise that they are being tested, which requires stimuli that closely resemble the real object or event that they simulate. For playback experiments, high quality recordings are therefore very essential, while the loudspeaker should not be visible but broadcasting from a plausible direction (see below, [45]).

\section{Natural experiments}

Fieldworkers often struggle with the fact that evolutionarily important events, such as encounters with predators, are rare or difficult to observe. The problem can be addressed by what is sometimes called a 'natural experiment'. This is not a true experimental manipulation but an intervention conducted to produce a systematic and large dataset. Strictly speaking, natural experiments are just observational studies as they lack the controls of a randomised experiment.

An early, dramatic example for an natural experiment is Kortlandt's report of antipredator behaviour by wild chimpanzees involving cooperative defence and the use of weapons, triggered by presenting a mobile stuffed leopard [46]*. A related more recent example is a study with free-ranging leopards to investigate primate alarm calling behaviour [47]. Here, the 'natural experiment' consisted of capturing and radio-tagging wild leopards in Tai Forest, Ivory Coast, and following them through the forest to monitor their hunting behaviour and the primates' corresponding anti-predator responses (fig. 1). One finding was that leopards spent extended periods of time hiding in the vicinity of monkey groups, but moved on as soon as detected by the monkeys -- valuable information that would have been extremely difficult to obtain with naturalistic observations alone.

-- Figure 1 --

\section{Controlled experiments}

Habituating wild primates to human observers is a lengthy, difficult and often costly process, which can take several years [48]. As a result, primate fieldwork is almost always plagued by small sample size, to the effect that within-subject designs are often the only possibility. Between-subjects designs are also possible, for example if a large number of primate groups are available for sampling [49]. The advantage is that each group can only be tested once, which removes concerns about dependent data. A recent example for a within-subjects design is a study with five putty-nosed 
monkey females. This monkey species is interesting because adult males combine two vocalisations, pyows and hacks, into different call sequences that are linked to specific external events [50]*. Series consisting of 'pyows' are a common response to leopards, while 'hacks' or 'hacks' followed by 'pyows' are regularly given to crowned eagles. However, males also produce sequences consisting of several 'pyows' followed by several 'hacks', which reliably predicts forthcoming group progression, regardless of context [51], [52]. In playback experiments, the females' reactions to their own male's 'pyows', 'hacks' and 'pyow-hack' sequences were tested. It was found that the subjects started group progressions after hearing 'pyowhack' sequences and also responded appropriately to the other call series [53]. This within-subjects design revealed that, in this primate, meaning is encoded at the level of call sequences, not just the individual calls, despite a very small number of subjects (fig. 2).

-- Figure 2 --

\section{Complex designs}

Sound playbacks have also been used in more elaborate designs, for instance in combination with real-life events or to in order to simulate two separate events. A recent example is a study on triadic awareness in chimpanzees [54]. Here, a subject was followed until a real agonistic interaction occurred with another group member. Provided no reconciliation took place, the researchers then continued to follow the subject and, after an average of two hours, they played back the aggressive barks of an ally (or non-ally) of their former opponent (fig. 3). Subjects responded in ways that suggested they were aware of the social relationships of other group members, i.e., who was likely to support whom, regardless of genetic ties.

-- Figure 3 --

An example of a two-events design is a playback study on mate guarding behaviour in baboons [55]. In this species, dominant males try to monopolise access to oestrous females to form 'consortships'. In the experiment, subjects heard a consorting male's grunts and an oestrous female's copulation calls in close succession but from two different speakers positioned 40m apart. This suggested to listeners that the male and female had separated and that she was now copulating with another male. As predicted, subjects reacted more strongly to this situation compared to when the female's copulation calls were combined with the grunts of a non-consorting or a formerly consorting male.

Two-events designs have also been used to simulate rank reversal in social groups [56]**, [57] and temporal separation between events [34]. For example, in putty-nosed monkeys, two disturbances (eagle attack or falling tree) were combined with male alarm calls about 30s later [58]. Results showed that listeners considered the previous contextual information as the likely cause for the subsequent alarm calls, suggesting that pragmatic factors are important in call interpretation in primates. An important type of two-event design is the 'habituation-dishabituation' experiment. Here, the goal is to study the effects of experience with one type of stimulus on how subjects respond to a second type of stimulus. These designs are suitable, for example, to investigate perceptual discrimination [22], psychoacoustics [59], or the conceptual organisation underlying communication [33]. 


\section{Visual stimuli}

Although playbacks are probably the most popular type of field experiments, they are by no means the only ones. In the early days of primatology, researchers carried out experiments by capturing and translocating live animals [60]*. Nowadays, this is usually done by broadcasting vocalisations, mainly because of the stress caused by capturing and moving animals between social groups.

Field experiments can also be based on the systematic presentation of objects with specific significance, such as food, predators, conspecifics or tools. Visual stimuli have been used to study economic decision-making [61], social learning [62], individual learning [63], cultural behaviour [64]; [65], or decision-making [66]. Predator presentations also have a long history in primate fieldwork [46], for instance to study anti-predator behaviour, mixed-species grouping or cognition [67], [68]*, [69], [70], [71], [51]. Finally, object presentations can be particularly useful in studies of tool use, with a large number of examples in the recent literature, especially on chimpanzees and capuchin monkeys [72], [73], [74].

\section{Experimenting with intelligent species}

Much scientific progress has been made in the last 40 years since field playbacks have been introduced to primatology. At the same time, however, researchers working with great apes have tended to shy away from field experiments, and it is interesting to explore the underlying reasons. One concern has to do with the advanced mental capacities of great apes, and the related question of whether it is possible (and morally acceptable) to produce meaningful results by interfering with their natural lives. Indeed, recent experience has shown that one great difficulty of working experimentally with great apes is to create plausible and credible situations, so that subjects do not realise that they are being tested, as is routinely done in human social psychology.

Some of the first field experiments with great apes have been in relation to long-distance calls, which are relatively easy to carry out (orang-utans [16]; chimpanzees [75], [76]), but it has long remained controversial whether playbacks are equally suitable to study close-range communication and cognition. A first attempt was a study by Slocombe et al [77]**, who tested whether chimpanzees could discriminate between different types of screams. The study is mainly relevant for its methodological advance because it illustrates, in great detail, the conditions that have to be in place for a successful playback experiment with socially highly aware animals (fig. 4).

\section{-- Figure 4 --}

Another reason why researchers of great apes have avoided field experiments is the possibility that experimental manipulations may have unwanted long-term effects. For example, Clarke et al. [78] found that Lar gibbons showed long-term behavioural effects hours after exposure to a predator model. A sensible way to avoid negative consequences is to work with stimuli that already occur regularly in the animals' natural lives. For example, although it is possible to simulate rank reversals in primates [56]**, designing such an experiment that involves the alpha male of a 
chimpanzee group may be ethically irresponsible. Changes in the alpha position can have severe, sometimes violent, implications for other group members, suggesting that this topic should not be investigated experimentally. This example again highlights the fact that playback experiments need to be grounded in careful, longterm behavioural observations and a profound knowledge of the study animals in order to be effective and appropriate.

Another difficulty with working with great apes is that administering experimental trials is often extremely time-consuming, necessitating careful planning. For this reason alone, the number of trials per individual needs to be kept to a minimum, ideally to one trial per condition. Retesting subjects with the same stimulus can be problematic, due to unwanted learning effects across trials. But sometimes this is necessary, for example if responses are subtle or to capture important intraindividual variation. To determine the maximally acceptable exposure rate per individual, a sensible approach is to determine the mean rate at which a test stimulus occurs under natural conditions. These principles have recently been applied to study the cognition, communication and culture of the Sonso chimpanzee community in Budongo Forest, which led to some progress regarding their cognitive capacities underlying communication and culture [50,52-54,64,67,68,72,79-81].

\section{Ethics}

Field experiments are an intrusion into the natural life of an animal and this can raise ethical issues. Some primate groups may contain vulnerable members or are ecologically challenged, so that researchers may decide against experimental interventions. For example, it may be unwise to induce anti-predator behaviour in mothers with newborn offspring, who may accidentally harm them during escape or be prevented from feeding. Some interventions, such as providing food, can facilitate disease transmission, alter the feeding habits of subjects or change their relationship to humans. Some populations are threatened by extinction, which may suggest that conservation research should be prioritised over basic research.

It is essential that researchers consider carefully whether a planned experimental intervention may be harmful and could have adverse long-term effects. Predation, aggression and intergroup encounters are natural components of a primate's life and there is no reason these topics should not be investigated in a scientifically rigorous way. However, it is good practice to have field experiments assessed by an independent ethics review board, which has the necessary expertise, so that adequate ethical standards are guaranteed. This is also in the self-interest of the researcher as it provides protection from judgement errors and is often a precondition for publication.

\section{Conclusions}

Field experiments are a powerful tool to study primate behaviour. Various procedures have been developed over the years, but virtually all depend on a robust understanding of the natural behaviour patterns of the study animals. As such, field experiments are usually the final step in a research programme, to test the causality of hypotheses obtained from observational studies. The most common techniques involve simulating the presence and behaviour of other individuals, by playing back 
their vocalisations, but object presentations have also been used in various ways.

Careful planning and piloting is almost always essential and researchers are advised to have their plans reviewed by an ethics board. A good strategy is to develop an experimental design that allows test stimuli to be blended into the subject's natural environment. This way, field experiments are likely to create responses that are evolutionarily relevant, socially meaningful and theoretically interpretable.

\section{Acknowledgement}

This project has received funding from the European Union's Seventh Framework Programme for research, technological development and demonstration under grant agreement no 283871. I am grateful to Tecumseh Fitch for valuable advice in revising this manuscript.

\section{References}

1. Wallman J: Aping language. New York: CUP; 1992.

2. Carpenter CR: A field study of the behavior and social relations of howling monkeys. Comp. Psychol. Monog. 1934, 10.

* one of the first systematic field studies with non-human primates

3. Struhsaker TT: Auditory communication among vervet monkeys (Cercopithecus aethiops). In Social communication among primates. Edited by Altmann SA: University of Chicago Press; 1967:281-324.

4. Waser PM: Experimental playbacks show vocal mediation of intergroup avoidance in a forest monkey. Nature 1975, 255:56-58.

* first demonstration that playback experiments can be used in primate fieldwork to address important biological questions

5. Unknown: Can monkeys talk? In Daily Alta California. Edited by; 1890. vol 83.]

6. Seyfarth RM, Cheney DL, Marler P: Monkey responses to three different alarm calls: Evidence of predator classification and semantic communication. Science 1980, 210:801-803.

* first experimental evidence for language-like capacities in wild primates

7. Cheney DL, Seyfarth RM: How monkeys see the world: Inside the mind of another species. Chicago: Chicago University Press; 1990.

** inspiring review of experimental work on the cognitive capacities of wild primates

8. Cheney DL, Seyfarth RM: Vocal recognition in free-ranging vervet monkeys. Animal Behaviour 1980, 28:362-367.

9. Cheney DL, Seyfarth RM: How vervet monkeys perceive their grunts: field playback experiments. Animal Behaviour 1982, 30:739-751.

10. Seyfarth RM, Cheney DL: Grooming, alliances and reciprocal altruism in vervet monkeys. Nature 1984, 308:541-542.

11. Cheney DL, Seyfarth RM: Assessment of meaning and the detection of unreliable signals by vervet monkeys. Animal Behaviour 1988, 36:477-486.

12. Robinson JG: Vocal regulation of inter- and intragroup spacing during boundary encounters in the titi monkey, Callicebus moloch. Primates 1981, 22:161-172.

13. Mitani JC: Responses of Gibbons (Hylobates-Muelleri) to Self, Neighbor, and Stranger Song Duets. International Journal of Primatology 1985, 6:193-200. 
14. Whitehead JM: The effect of the location of a simulated intruder on responses to long-distance vocalizations of mantled howling monkeys (Alouatta palliata palliata). Behaviour 1989, 108:73-103.

15. Tenaza RR: Intergroup calls of male pig-tailed langurs (Simias concolor). Primates 1989, 30:199-206.

16. Mitani JC: Sexual selection and adult male orangutan long calls. Animal Behaviour 1985, 33:272-283.

17. Mitani JC: The behavioral regulation of monogamy in gibbons (Hylobates muelleri). Behav. Ecol. Sociobiol. 1984, 15:225-229.

18. Mitani JC: Gibbon song duets and intergroup spacing. Behaviour 1985, 82:5996.

19. Raemaekers J, Raemaekers P: The ooaa duet of the gibbon (Hylobates lar) - a group call which triggers other groups to respond in kind. Folia Primatologica 1984, 42:209-215.

20. Semple S: The function of Barbary macaque copulation calls. Proc. Roy. Soc. Lond. B. 1998, 265:287-291.

21. Masataka N: Psycholinguistic analyses of alarm calls of Japanese monkeys (Macaca fuscata fuscata). Am. J. Primatol 1983, 5:111-125.

22. Fischer J: Barbary macaques categorize shrill barks into two call types. Animal Behaviour 1998, 55:799-807.

23. Sugiura $\mathrm{H}$ : Temporal and acoustic correlates in vocal exchange of coo calls in Japanese macaques. Behaviour 1993, 124:207-225.

24. Macedonia JM: What is communicated in the antipredator calls of lemurs: evidence from playback experiments with ring-tailed and ruffed lemurs. Ethology 1990, 86:177-190.

25. Oda R, Masataka N: Interspecific responses of ringtailed lemurs to playback of antipredator alarm calls given by Verreaux's sifakas. Ethology 1996, 102:441-453.

26. Zuberbühler K, Noë R, Seyfarth RM: Diana monkey long-distance calls: messages for conspecifics and predators. Animal Behaviour 1997, 53:589604.

27. Fischer J, Hammerschmidt K, Todt D: Factors affecting acoustic variation in barbary macaque (Macaca sylvanus) disturbance calls. Ethology 1995, 101:51-66.

28. Ramakrishnan U, Coss RG: Age differences in the responses to adult and juvenile alarm calls by bonnet macaques (Macaca radiata). Ethology 2000, 106:131-144.

29. Chapman CA, Chapman LJ: Mixed-species primate groups in the Kibale Forest: Ecological constraints on association. Int. J. Primatol. 1996, 17:3150.

30. Noë R, Bshary R: The formation of red colobus diana monkey associations under predation pressure from chimpanzees. Proc. Roy. Soc. Lond., Series B 1997, 264:253-259.

31. Treves A: Has predation shaped the social systems of arboreal primates? International Journal of Primatology 1999, 20:35-67.

32. Cheney D, Seyfarth R, Silk J: The responses of female baboons (Papio cynocephalus ursinus) to anomalous social interactions: Evidence for causal reasoning? Journal of Comparative Psychology 1995, 109:134-141.

33. Zuberbühler K, Cheney DL, Seyfarth RM: Conceptual semantics in a nonhuman primate. Journal of Comparative Psychology 1999, 113:33-42. 
34. Zuberbühler K: Causal cognition in a non-human primate: field playback experiments with Diana monkeys. Cognition 2000, 76:195-207.

35. Zuberbühler K: Causal knowledge of predators' behaviour in wild Diana monkeys. Animal Behaviour 2000, 59:209-220.

36. Hammerschmidt K, Fischer J: Maternal discrimination of offspring vocalizations in Barbary macaques (Macaca sylvanus). Primates 1998, 39:231-236.

37. Rendall D, Rodman PS, Emond RE: Vocal recognition of individuals and kin in free-ranging rhesus monkeys. Animal Behaviour 1996, 51:1007-1015.

38. Rendall D, Owren M, Rodman P: The role of vocal tract filtering in identity cueing in rhesus monkey (Macaca mulatta) vocalizations. Journal of the Acoustical Society of America 1998, 103:602-614.

39. Teixidor P, Byrne R: Can spider monkeys (Ateles geoffroyi) discriminate vocalizations of familiar individuals and strangers? Folia Primatologica 1997, 68:254-264.

40. Teixidor P, Byrne RW: The 'whinny' of spider monkeys: Individual recognition before situational meaning. Behaviour 1999, 136:279-308.

41. Olupot W, Waser P, Chapman C: Fruit finding by mangabeys (Lophocebus albigena): Are monitoring of fig trees and use of sympatric frugivore calls possible strategies? Int. J. Primatol. 1998, 19:339-353.

42. Muroyama Y, Thierry B: Species differences of male loud calls and their perception in Sulawesi macaques. Primates 1998, 39:115-126.

43. Zuberbühler K: Interspecific semantic communication in two forest monkeys. Proceedings of the Royal Society of London Series B-Biological Sciences 2000, 267:713-718.

44. Altmann J: Observational study of behavior: Sampling methods. Behaviour 1974, 49:227-267.

45. Zuberbühler K, Wittig RM: Field experiments with nonhuman primates: a tutorial. In Field and Laboratory Methods in Primatology: A Practical Guide. Edited by Setchell JM, Curtis DJ: Cambridge University Press; 2011:207-224.

46. Kortlandt A, Kooij M: Protohominid behaviour in primates. Symposium of the Zoological Society of London 1963, 10:61-88.

* early example of a natural experiment

47. Zuberbühler K, Jenny D, Bshary R: The predator deterrence function of primate alarm calls. Ethology 1999, 105:477-490.

48. Samuni L, Mundry R, Terkel J, Zuberbuhler K, Hobaiter C: Socially learned habituation to human observers in wild chimpanzees. Animal Cognition 2014:1-9.

49. Zuberbühler K: Referential labelling in Diana monkeys. Animal Behaviour 2000, 59:917-927.

50. Arnold K, Zuberbühler K: Language evolution: semantic combinations in primate calls. Nature 2006, 441:303.

* first evidence of a sequence-based semantic signal in non-human primates

51. Arnold K, Pohlner Y, Zuberbühler K: A forest monkey's alarm call series to predator models. Behavioral Ecology and Sociobiology 2008, 62:549-559.

52. Arnold K, Zuberbuhler K: Call combinations in monkeys: Compositional or idiomatic expressions? Brain and Language 2012, 120:303-309.

53. Arnold K, Zuberbühler K: Meaningful call combinations in a non-human primate. Current Biology 2008, 18:R202-R203. 
54. Wittig RM, Crockford C, Langergraber KE, Zuberbuhler K: Triadic social interactions operate across time: a field experiment with wild chimpanzees. Proceedings of the Royal Society B-Biological Sciences 2014, 281.

55. Crockford C, Wittig RM, Seyfarth RM, Cheney DL: Baboons eavesdrop to deduce mating opportunities. Animal Behaviour 2007, 73:885-890.

56. Bergman TJ, Beehner JC, Cheney DL, Seyfarth RM: Hierarchical classification by rank and kinship in baboons. Science 2003, 302:1234-1236.

** sophisticated experimental design based on simulating social interactions between two individuals

57. Slocombe KE, Kaller T, Call J, Zuberbuhler K: Chimpanzees Extract Social Information from Agonistic Screams. Plos One 2010, 5.

58. Arnold K, Zuberbuhler K: Female Putty-Nosed Monkeys Use Experimentally Altered Contextual Information to Disambiguate the Cause of Male Alarm Calls. Plos One 2013, 8.

59. Fitch WT, Fritz JB: Rhesus macaques spontaneously perceive formants in conspecific vocalizations. Journal of the Acoustical Society of America 2006, 120:2132-2141.

60. Kummer H, Gotz W, Angst W: Triadic differentiation: An inhibitory process protecting pair bonds in baboons. Behaviour 1974, 49:62-87.

* pinoneering field experiment with wild primates

61. Fruteau C, Voelkl B, van Damme E, Noe R: Supply and demand determine the market value of food providers in wild vervet monkeys. Proceedings of the National Academy of Sciences of the United States of America 2009, 106:12007-12012.

62. van de Waal E, Borgeaud C, Whiten A: Potent Social Learning and Conformity Shape a Wild Primate's Foraging Decisions. Science 2013, 340:483-485.

63. Pesendorfer MB, Gunhold T, Schiel N, Souto A, Huber L, Range F: The Maintenance of Traditions in Marmosets: Individual Habit, Not Social Conformity? A Field Experiment. Plos One 2009, 4.

64. Gruber T, Muller MN, Strimling P, Wrangham RW, Zuberbühler K: Wild chimpanzees rely on their cultural knowledge to solve an experimental honey acquisition task. Current Biology 2009, 19:1806-1810.

65. Kawai M: Newly-acquired pre-cultural behavior of the natural troop of Japanese monkeys on Koshima islet. Primates 1965, 6:1-30.

66. Menzel CR: Cognitive aspects of foraging in Japanese monkeys. Animal Behaviour 1991, 41:397-402.

67. Schel AM, Townsend SW, Machanda Z, Zuberbuhler K, Slocombe KE: Chimpanzee Alarm Call Production Meets Key Criteria for Intentionality. Plos One 2013, 8.

68. Crockford C, Wittig RM, Mundry R, Zuberbuhler K: Wild Chimpanzees Inform Ignorant Group Members of Danger. Current Biology 2012, 22:142-146.

* experimental demonstration that call production in chimpanzees is based on high levels of audience awareness

69. Casar C, Zuberbuhler K, Young RJ, Byrne RW: Titi monkey call sequences vary with predator location and type. Biology Letters 2013, 9.

70. Wich SA, de Vries H: Male monkeys remember which group members have given alarm calls. Proceedings of the Royal Society B-Biological Sciences 2006, 273:735-740. 
71. Ouattara K, Lemasson A, Zuberbühler K: Anti-predator strategies of freeranging Campbell's monkeys. Behaviour 2009, 146:1687-1708.

72. Gruber T, Muller MN, Reynolds V, Wrangham R, Zuberbuhler K: Communityspecific evaluation of tool affordances in wild chimpanzees. Scientific Reports 2011, 1.

73. Matsuzawa T: Field experiments on use of stone tools by chimpanzees in the wild. In Chimpanzee cultures. Edited by Wrangham RW, McGrew WC, deWaal FBM, Heltne PG; 1994:351-370.

74. Visalberghi E, Addessi E, Truppa V, Spagnoletti N, Ottoni E, Izar P, Fragaszy D: Selection of Effective Stone Tools by Wild Bearded Capuchin Monkeys. Current Biology 2009, 19:213-217.

75. Wilson ML, Hauser MD, Wrangham RW: Does participation in intergroup conflict depend on numerical assessment, range location, or rank for wild chimpanzees? Animal Behaviour 2001, 61:1203-1216.

76. Herbinger I, Papworth S, Boesch C, Zuberbuhler K: Vocal, gestural and locomotor responses of wild chimpanzees to familiar and unfamiliar intruders: a playback study. Animal Behaviour 2009, 78:1389-1396.

77. Slocombe KE, Townsend SW, Zuberbühler K: Wild chimpanzees (Pan troglodytes schweinfurthii) distinguish between different scream types: evidence from a playback study. Animal Cognition 2009, 12:441-449.

** first study to demonstrate that playbacks can be used to study intra-group communication in great apes

78. Clarke E, Reichard U, Zuberbühler K: The anti-predator behaviour of wild white-handed gibbons (Hylobates lar). Behavioral Ecology and Sociobiology 2012, 66:85-96.

79. Schel AM, Machanda Z, Townsend SW, Zuberbuhler K, Slocombe KE: Chimpanzee food calls are directed at specific individuals. Animal Behaviour 2013, 86:955-965.

80. Gruber T, Reynolds V, Zuberbuhler K: The knows and unknowns of chimpanzee culture. Communicative \& Integrative Biology 2010, 3:1-3.

81. Gruber T, Zuberbuhler K: Evolutionary origins of the human cultural mind. Psychologist 2012, 25:364-368. 